\title{
Analyzing the Increase of Network Life Time for Wireless Sensor Network with Smart Local Moving Algorithm using NS2 Simulator
}

\author{
Nakka Thirupathi Rao ${ }^{1}$, Debnath Bhattacharyya ${ }^{1}$ and Hye-jin Kim ${ }^{2}$ \\ ${ }^{1}$ Department of Computer Science \& Engineering Vignan's Institute of \\ Information Technology, Visakhapatnam-530049, India \\ ${ }^{2}$ Business Administration Research Institute, Sungshin W. University, \\ ${ }^{2}$ Bomun-ro 34da-gil, Seongbuk-gu, Seoul \\ nakkathiru@gmail.com,debnathb@gmail.com \\ hyejinaa@daum.net \\ (Corresponding Author)
}

\begin{abstract}
Energy economical routing could be a one in the entire key trusty space in Wireless sensing element Networks. The wireless sensing element network comprising of an oversized variety of sensing element nodes that has restricted energy store. The sensing element nodes area unit working with the help of the sources likes the battery. The saving of very useful resource like energy becomes supplementary a very significant subject in WSNs. The routing algorithms guarantee the conception of energy saving while not moving the standards of the network performance qualities like turnout, finish to finish Delay, operating cost and magnitude relation of Packet Delivery. In the existing system, the improved EEEMR Protocol is enforced with clustering algorithm. But, in the proposed model of the system we considered the SLM algorithm with the combination of EEEMR protocol and implemented the considered network model. The performance of the model considered was analyzed by considering various network performances analyzing metrics. By analyzing these considered metrics, the performance of the model can be understood and explained in detail. During this paper, we tend to area unit implementing sensible native Moving algorithmic program in EEEMR Protocol. The event of cluster primarily based sensing element networks cover in recent times exposed to reduce the congestion in networks, system delay, operating cost and increase the system turnout and packet delivery magnitude relation. Some graphical representation was observed in the section of results for the comparison of the both the systems considered in terms of the various network performance metrics. The currently considered network model was expected to be increase the life time of the network with the incorporation of the EEEMR protocol with new SLM algorithm instead of the EEEMR protocol with the combination of clustering algorithm. The performance was studied by using the simulation on NS2 simulator and results shows that the projected system is better than the prevailing system. The projected system energy utilization is diminished when compared to the prevailing system.
\end{abstract}

Keywords: Network Life Time, Wireless sensor network, EEEMR protocol, SLM algorithm, Throughput, Energy efficiency

Received (June 14, 2017), Review Result (August 11, 2017), Accepted (September 1, 2017) 


\section{Introduction}

The succession of wireless sensor detector networks is at first encouraged by various set of military applications. These networks are also utilized in various civilian application areas like detective work, observing the movement of enemies at borders of the countries and also help in ident6ifying the intruders from various sources to the unwanted or restricted areas in the country. They can also be useful for various sources like the chemical and other types of devices or the practical applied things which can be used for the chemical uses or for the practical applications or for the preparation of various materials or devices with the help of these chemical units. They can also be used for tracking the position of vehicles, cars, buses for public transportation systems, security of the vehicles and also for the monitoring of the images with various applications. The best application they can be used was the health care applications.

The sensor networks can be used in the case of both the wired and for the wireless networks. The wireless networks will consist of sometimes very little number of batteries for the supply of energy and in some cases, the devices may require the very huge number fop batteries for supplying the energy source for the working of very huge network. The number of batteries present in a device will depend on the amount of energy the system has to provide for executing the task that was being given to that system. In some cases the devices will work with the combination of the two or more systems or units such that to perform the task given or the task for which the device was intended to be developed for. Every node in the sensor unit or the device will consist of very little number of nodes in some cases and in some other cases, we can find a very huge number of units for performing the task. The nodes in the network will consists of the features like the sensing or identifying the type of the device or the measurement of the physical quantities like temperature, weight etc.

These devices can also be used for the measurement of finding the values of the various performance measures of the systems like the speed of the vehicles, temperature available various places like remote places the human cannot be able to enter and also to measure the pressure that was available at various corners of the devices or located at various locations of the units. These also can be used for various units like the flights and the aerial devices. These devices can also be used for the measurement of the light or the amount of light available at various places like rooms or at various places where the presence or entrance for the humans is very difficult. The structure of the nodes that were being used in the ad-hoc sensor networks can be used for various applications. Due to the movement of the nodes in the network, the network structure might change from time to time. As a result of this phenomenon, the performance of the network at various intervals of times, we can observe the changes at all these places. The performance can also be measured as the nodes changes their locations, the bandwidth and the receiving capacity of the signal may also change from time to time. The size of the system was very less due to the presence of various sensors and the size of these sensors also very less.

The energy consumption of the system is very important consideration for the system. As the size of the device is less and the device can be placed at various locations. But, it is not always possible to set power backup or charge the device from time to time. Hence, the power consumption or the energy usage or the energy consumption can be taken as one of the most important consideration for the system. Hence, it is always not possible for the users to replace the battery from time to time or to recharge the battery or the power source. The increasing the usage of the network from time to time has to be increased otherwise, the system cannot be used for most of the cases or most of the users. By increasing these factors or considering these points, the life time of the network or the survival time of the network can be increased in the sense of working or working with less usage of the power or the battery source. 


\section{Proposed Work}

The usage of the wireless sensor network for various applications and also in various scenarios can be considered based on the points like the advantages and the better opportunities or the best offers that we are getting from these sort of networks. Our main goal in the design of these scenarios was to increase the life time of the network that means the working time of the network must be increased such that the more number of users or more number of applications can be used or being used by this sort of sensor network. Hence, our motto is to identify the implementation of the proposed scenario system with the help of EEEMR protocol. But, in the previous works, the EEEMR protocol was being used already several users with the combination of the clustering algorithm and tried to analyze the behavior of the system. They also tried to analyze and estimate the performance of the network at various scenarios with various set of observations. The existing work in the area of this topic was done and the results show that the considered system or the model was observing more energy consumption and fewer throughputs from the network.

In order to steady the performance of the system and also to achieve the better performance by the considered model of the network, we tried to implement the considered model with EEEMR protocol being used for the nodes and also incorporated the SLM algorithm to achieve the better results. Several characteristics of the considered system were studied such that the analysis of the system tried to understand. Several characteristics like the throughput of the network, packet delivery ratio and also some other important parameters studied and analyzed for the both scenarios. The implementation part for the proposed system with proposed model was studied and presented in the following sections.

\section{Smart Local Moving Algorithm}

The Smart local moving algorithm not only identifies the structure of the network or the system but also it identifies the solution for the units region wise for the best solutions and also for the similarity or the relevance of the data in the networks such that to merge the best nodes and also for the node movements. The Smart local moving algorithm income by constructing a reduced network. The SLM algorithmic rule also will construct a reduced network, however before it will thus, it initial takes another steps. The SLM algorithmic rule iterates over all network within the gift network structure. In different cases, a network structure is obtained consisting of multiple networks that include a number of the nodes within the sub network. Once a network structure has been obtained for every of the sub networks, the SLM algorithmic rule constructs a reduced network.

The SLM algorithmic program then performs associate initial assignment of the nodes within the reduced network to network. Nodes corresponding with network within the same sub network square measure assigned to identical network within the reduced network. Hence, for every sub network, there's one network within the reduced third network. At this time, the whole method starts everywhere once more, however this point supported the reduced network instead of the initial one. During this approach, the SLM algorithmic program has a lot of freedom in looking for high-quality solutions to the modularity optimization downside. Parenthetically the SLM algorithmic program, we tend to once more contemplate our martial art club example. We tend to 1st return to work one. This figure shows the network structure obtained by applying the native moving heuristic within the original network.

There square measure six network, every indicated employing a completely different color. As explained higher than, when applying the native moving heuristic within the original network, for every network a sub network is built. Within the inexperienced, blue, purple, and yellow sub networks, this leads to all nodes being assigned to identical network. Nodes corresponding with network within the same sub network square measure 
initio assigned to identical network within the reduced network. As are often seen, nodes A1 and A2 within the reduced network have remained within the same network. In the next phase of the iteration, we tend to begin with nodes being allocated to the network which were gained or obtained from the first set of iteration. Within the third iteration, the network obtained within the second iteration square measure our place to begin, and so on. This happens once a network structure is obtained that can't be improved additional either by merging network or by moving individual nodes from one network to a different. Once running the SLM algorithmic program in associate repetitive approach, the algorithmic program keeps looking for prospects to extend modularity by network and by moving sets of nodes from one network to a different. Hence, within the case of the repetitive variant of the SLM algorithmic program, it's going to continuously be doable to get additional enhancements in network structure by acting a lot of iterations of the algorithmic program.

The major steps followed in the proposed algorithmic model of SLM algorithm and the EEEMR algorithm is as follows:

1. The algorithmic rule is largely separated to the quantity of number of rounds.

2. The primary nodes which are having the so good and best energy nodes square measure can be treated as a cluster head with every way for that exact cluster and transmission of knowledge is performed.

3. The calculation part of the node edge at that particular point is done by the cluster head at the beginning of the second around the cluster head calculates the remaining energy of the actual members.

4. Total number of cluster heads that were present in the network will perform the similar tasks with their cluster members and effective bunch is performed to achieve the bottom station by choosing best cluster head.

5. Each node in the network will calculate the edge worth. If the edge worth belonging to a node is bigger than the threshold value worth, the nodes are candidate for the cluster head of that cluster for successive spherical.

6. The threshold value of the cluster head value is below the edge worth of network, the pointed cluster head is removed or terminated and once more the cluster head choice method is performed in this cluster.

7. The value that was calculated for the cluster head is below the edge worth in this time the cluster member's square measure send their perceived knowledge to the closest cluster head. This method implementation or working will be considered continuously till the new cluster head is chosen in the selected group of the cluster.

8. The cluster heads which was identified as an best station or the best cluster head or the station can be used to transmit the data to the bottom station and don't involve base station to pick cluster head at every spherical and to scale back energy consumption at every spherical.

\section{Simulation Setup}

In the present work, we were intended to increase the life time working of a sensor network especially in the scenario of a wireless sensor network. The motto of us will be completed by choosing the best setup with the combination of best used equipment such that to increase the performance of the system by decreasing the energy usage of the system or the considered system. The decreasing of the energy consumption by the proposed system itself is nothing but the increase of the life time or working time of the system or the considered network. Hence, an attempt has been made such that to reduce the energy consumption by choosing the components of the network such that to use very less amount of energy. 
Several authors had used the EEEMR protocol in the networks such that to work with the combination of the clustering algorithm. The consumption was huge by $t$ eh considered systems with this combination.

Hence, we had considered this scenario and incorporated an clustering algorithm like the SLM algorithm with the combination of the EEEMR protocol such that to increase the life time of the system and also decrease the usage of the power to the system. As the considered scenario was somewhat difficult to build the system and the scenario, we had made an attempt to simulate the same configured problems with same configured techniques. We also tried to implement and establish the same environment such that in the real time scenarios. With the established model in the simulated setup, we had considered various performance metrics of the network such that to analyze the behavior of the network with our considered or proposed scenario. The results were observed and the values were presented in the form of graphical representation. The simulation setup was done and executed the total process in the simulator of NS-2 and the results were displayed in the form of graphical model in the results section.

1. Throughput: The number of packets being delivered successfully per second in the network from source to the destination.

2. End to end delay: This delay can be calculated as the time taken by the packets from source to the destination over a wireless sensor network.

3. Packet delivery ratio: This ratio can be calculated as the ratio of the packets being received to the packets being sent from the sender.

4. Operating cost: This cost can be calculated as the amount of energy we need to spend such that to perform the task that was being given to the network.

5. Energy: The amount of energy or the amount of power that can be used by the system or the network to perform the intended task to be performed by the proposed model of the network.

\section{Simulation Results}

We have done our exploration investigation in remote sensor arranges by utilizing NS2. Similar investigation done between proposed framework and existing framework. The Operating cost examination is appeared in Figure 2. Working expense is diminishes when contrasted with the current framework. In initial $40 \mathrm{~s}$ the current framework is superior to the proposed framework. On the off chance that the reproduction time is increment the proposed framework is superior to the current framework. The Delay examination is appeared in Figure 3. Delay is diminishes when contrasted with the current framework. In initial $40 \mathrm{~s}$ the current framework is superior to the proposed framework. In the event that the reproduction time is increment the proposed framework is superior to the current framework. The Throughput correlation is appeared in Figure 4. Throughput is increment when contrasted with the current framework. In initial $40 \mathrm{~s}$ the current framework is superior to the proposed framework. In the event that the recreation time is increment the proposed framework is superior to the current framework. The PDR correlation is appeared in Figure 5. PDR is increment when contrasted with the current framework. In initial $20 \mathrm{~s}$ the current framework is superior to the proposed framework. In the event that the reproduction time is increment the proposed framework is superior to the current framework. 


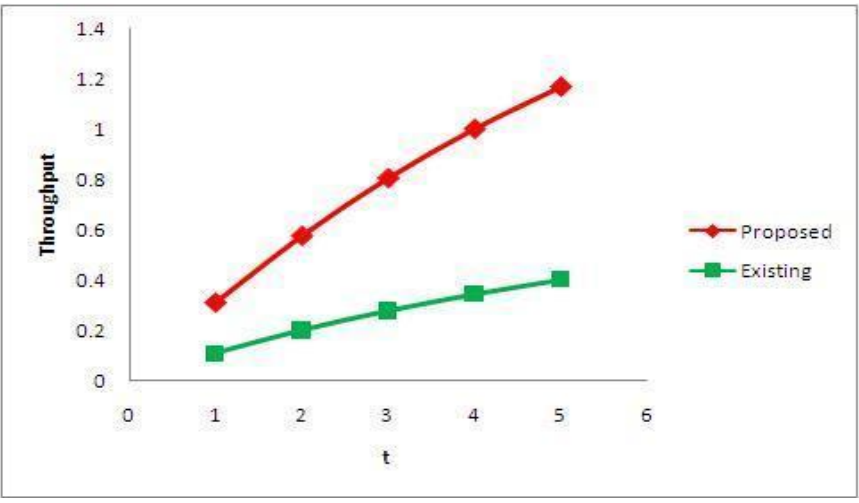

Figure 1. Throughput Vs Time (Sec)

In the above Figure 1, the relation between the time taken for the implementation part of the system with considered protocol and the considered algorithm was implemented. The consideration was done with respect to the throughput of the network model with respect to the change in time from time to time. The performance was done from the simulator model and the results were drawn and represented in the form of a graphical representation. From the graphical representation, it is clearly understood that the representation was done with respect to the time for both the algorithms. The existing algorithm considered here was the clustering algorithm and the proposed model of considered algorithm was the smart local moving algorithm. The observation is that the throughput of the proposed model was increasing as time increases than the existing algorithmic model. The throughput of the considered model, the number of packets being transmitting through the network for the considered time and for the considered algorithmic combination with EEEMR protocol was observed good for the proposed model when compared with the existing clustering algorithm model of the EEEMR protocol model.

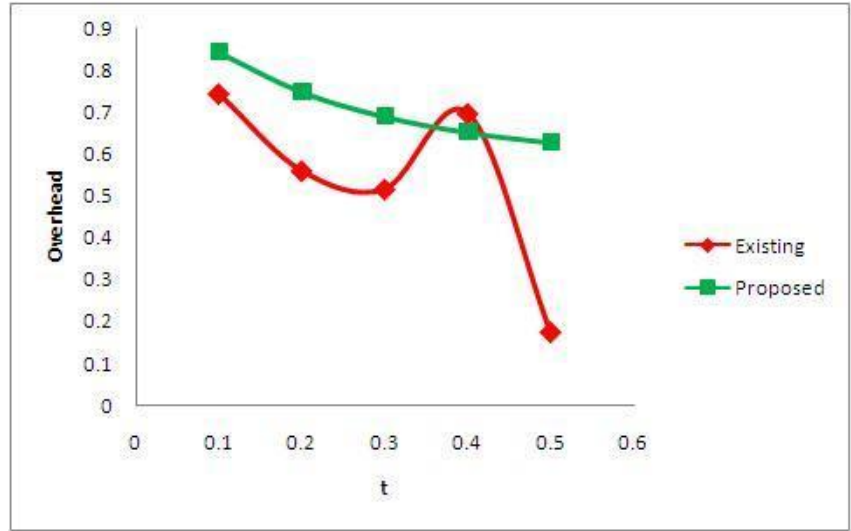

Figure 2. Operating Cost Vs Time (Sec)

In the above Figure 2, the relation between the time taken for the implementation part of the system with considered protocol and the considered algorithm was implemented. The consideration was done with respect to the operating cost of the network model with respect to the change in time from time to time. The performance was done from the simulator model and the results were drawn and represented in the form of a graphical representation. From the graphical representation, it is clearly understood that the representation was done with respect to the time for both the algorithms. The existing algorithm considered here was the clustering algorithm and the proposed model of considered algorithm was the smart local moving algorithm. The observation is that the 
operating cost of the proposed model was decreasing as time increases than the existing algorithmic model.

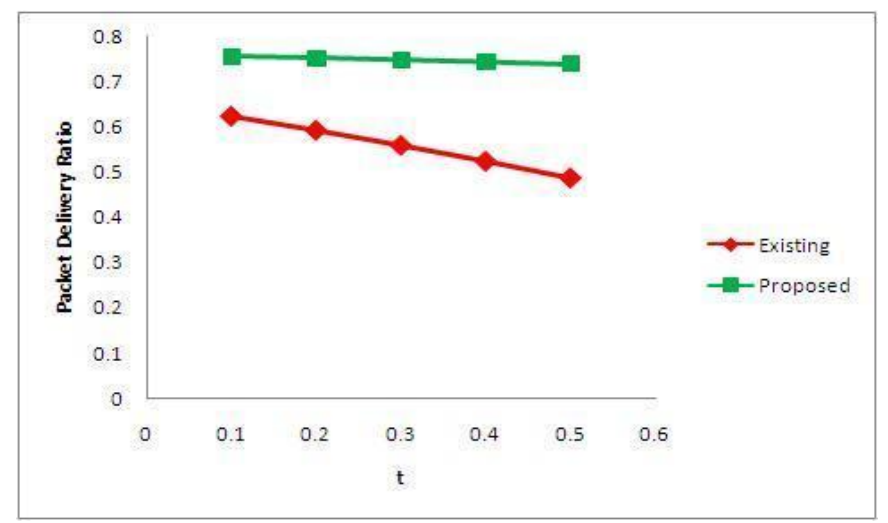

Figure 3. Packet Delivery Ratio Vs Time (Sec)

In the above Figure 3, the relation between the time taken for the implementation part of the system with considered protocol and the considered algorithm was implemented. The consideration was done with respect to the operating cost of the network model with respect to the change in time from time to time. The performance was done from the simulator model and the results were drawn and represented in the form of a graphical representation. From the graphical representation, it is clearly understood that the representation was done with respect to the time for both the algorithms. The existing algorithm considered here was the clustering algorithm and the proposed model of considered algorithm was the smart local moving algorithm. The observation is that the operating cost of the proposed model was decreasing as time increases than the existing algorithmic model. The packet delivery ratio for the considered system was the number of packets being delivered for the system from the sender to the receiver for the time considered by the proposed model system. The existing system performance was very less when compared to the system performance when the case of the proposed algorithm with EEEMR protocol was considered.

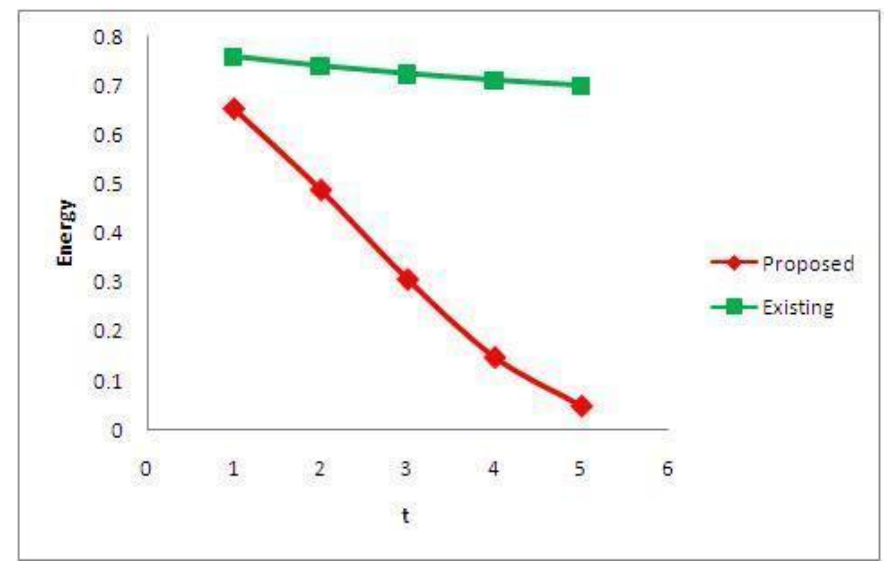

Figure 4. Energy Vs Time (Sec)

The consideration was done with respect to the operating cost of the network model with respect to the change in time from time to time. The performance was done from the simulator model and the results were drawn and represented in the form of a graphical representation. From the graphical representation, it is clearly understood that the representation was done with respect to the time for both the algorithms. The existing 
algorithm considered here was the clustering algorithm and the proposed model of considered algorithm was the smart local moving algorithm. The observation is that the energy utilization and consumption of the energy for the system was considered very much highly for the systems for the proposed model was decreasing as time increases than the existing algorithmic model. The energy consumption for the system is a very important constraint for the system in the performance of the considered model as it depends on the total functioning of the system. Hence, this consumption of the energy is considered as the important factor for analyzing the performance of the system. The packet delivery ratio for the considered system was the number of packets being delivered for the system from the sender to the receiver for the time considered by the proposed model system. The existing system performance was very less when compared to the system performance when the case of the proposed algorithm with EEEMR protocol was considered.

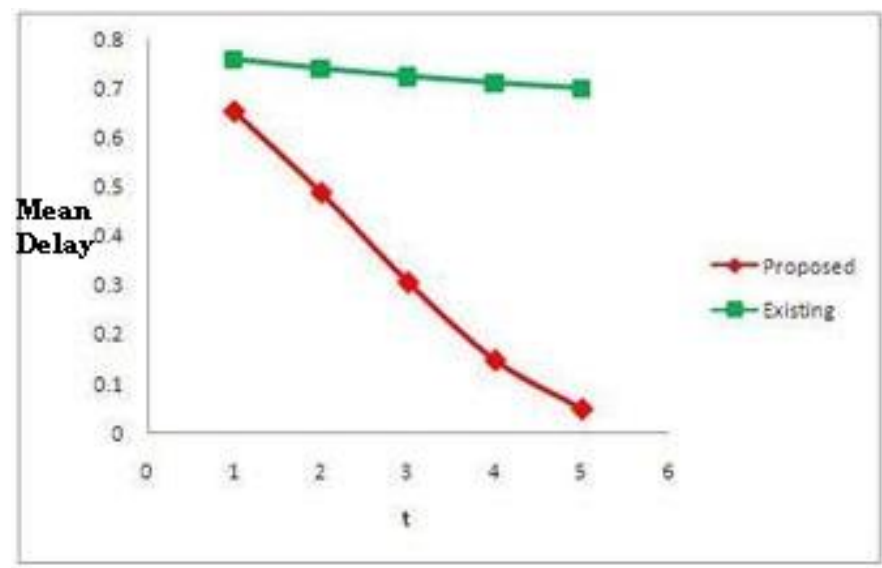

Figure 5. Mean Delay Vs Time (Sec)

The amount of time that is being taking by the proposed system for the implementation of the model considered with both the cases like the EEEMR protocol with clustering algorithm and also the EEEMR protocol with SLM algorithm. The method was studied for both the cases and the performance of the proposed model with the existing and proposed system was studied and analyzed. The requirement of the mean delay for the overall assessment of the system need to be analyzed such that to make the changes if any required or needed to make the model more stable more functional. The calculation part was done and the results were displayed in the form of some graphical representation.

Table 1. Details of the Parameters and Values used in the Existing and Proposed Models

\begin{tabular}{|l|l|}
\hline \multicolumn{1}{|c|}{ Parameter } & \multicolumn{1}{c|}{ Value } \\
\hline Protocols & EEEMR Protocol \\
\hline Algorithm used & Smart Local Moving Algorithm \\
\hline Size of the terrain & Terrain Size 1020 $\times 1020$ \\
\hline MAC Layer details & 802.11 \\
\hline Number of nodes used in the model & 400 \\
\hline $\begin{array}{l}\text { Type of the channel used in the } \\
\text { proposed model }\end{array}$ & Wireless Channel \\
\hline Model of the Antenna & Omni Antenna \\
\hline $\begin{array}{l}\text { Propagation Model used in the } \\
\text { considered model }\end{array}$ & Two Ray Ground \\
\hline
\end{tabular}




\begin{tabular}{|l|l|}
\hline Interface having the Queue Length & 75 \\
\hline Interface having Queue Type & Drop Tail/Pri Queue \\
\hline $\begin{array}{l}\text { Time taken for Simulation process } \\
\text { in seconds }\end{array}$ & $160 \mathrm{~s}$ \\
\hline Simulator & NS-2.34 \\
\hline
\end{tabular}

\section{Conclusion}

In the current paper, by using the NS-2 simulator we tried to find the various parameters of the wireless sensor network such that to analyze and increase the working life time of the network model. Several performance metrics of the wireless sensor network like the throughput of the network, delay of the network, packet delivery ratio of the network model, overhead or the operating cost of the network in terms of cost, energy, usage of the sources like the power and energy was also being calculated and also presented in the form of various graphical representation. In the existing work, we had discussed the implementation of EEEMR protocol with the combination of clustering algorithm. We had implemented the both the combination of the protocol with two clustering model algorithms. We had implemented the EEEMR protocol with the combination of the SLM algorithm, the other clustering algorithm with respect to the proposed network model. The total analysis and setup was observed and created in the NS-2 simulator with the help of the programming the simulator. The two combinations of the algorithms were being implemented with the help of the EEEMR protocol such that the analysis can be understood clearly. The main motto of us to achieve the working of the proposed network model should work better in the case of the energy consumption that is the network should function by consuming very less amount of energy such that the working life time of the model could be performed well.

Hence, a comparison was performed ion the results section in the case of all the performance metrics. The throughput was good for the model. The energy consumption was very good and the execution was very good for the proposed model when compared with the existing model of the algorithmic models. With the help of the proposed model of the system, we are trying to advance the quality of service parameters like Delay, Operating cost, mean delay, throughput, Packet Delivery Ratio and Energy of wireless sensor networks. The throughput of the considered model was increased in a gradual range of nearly around $45 \%$ increase when compared to the existing model of the system. The important characteristics are Packet Delivery Ratio increase around a value of $23 \%$ when compared to the previous model. The delay is decreased to a range of around $35 \%$ decrease and the Operating cost is around 40\% decreases. And the important characteristic is the energy consumption which is decreased in a fruitful way of around $13 \%$ in which the previous one is around $25 \%$. Hence, by observing the performance of all these metrics, the life time of the network and also the quality of service being provided to the end users by using this sort of techniques.

\section{References}

[1] O. Jumira, "Energy Efficiency in Wireless Networks", Wiley, (2013).

[2] T. Zhang and J. Cao, "A Small World Network Model for Energy Efficient Wireless Networks", IEEE Communications Letters, vol. 17, (2013), pp. 1928-1931.

[3] S. P. Singh, "A Survey on Cluster Based Routing Protocols in Wireless Sensor Network", International Conference on Advanced Computing Technologies and Applications, (2015).

[4] P. M. Dave and P. D. Dala, "Simulation and Performance Evaluation of Routing Protocols in Wireless Sensor Network", International Journal of Advanced Research in Computer Communication Engineering, vol. 2, (2013), pp. 1405-1412.

[5] Y. Gu and Q. Wu, "On Efficient Deployment of High-end Sensors in Large-scale Heterogeneous WSNs", IEEE 6th International Conference on Mobile Adhoc and Sensor Systems, (2009). 
[6] Z. Aliouat and S. Harous, "An Efficient Clustering Protocol Increasing Wireless Sensor Networks Lifetime", International Conference on Communication, Networking \& Broadcasting, (2012).

[7] G. Sharma, "Hybrid Sensor Networks: A Small World," 6th ACM International Symposium on Mobile ad hoc Networking and Computing, (2005).

[8] O. Younis and S. Fahmy, "Distributed Clustering in ad-hoc Sensor Networks: A Hybrid, Energyefficient Approach", Twenty-Third Annual Joint Conference of the IEEE Computer and Communications Societies, INFOCOM, (2004).

[9] E. Akhtarkavan, "Clustering Algorithm to Reduce Power Consumption in Wireless Sensor Network", ACSIJ, (2014)

[10] A. E. Kamal, "Routing Techniques in WSN: A Survey", IEEE Wireless Communications, (2004), pp. 628.

[11] J. A. Nayak, "Improving the Network Life Time of Wireless Sensor Network using EEEMR Protocol with Clustering Algorithm”, IJSNDC, vol. 6, no. 1, (2017), pp. 2-4. 